J. Numer. Anal. Meth. Geomech., 25, (2001), 1285-1303

\title{
DETERMINATION OF ROCK MASS STRENGTH PROPERTIES BY HOMOGENIZATION
}

\author{
A. POUYA AND M. GHOREYCHI \\ G.3S - Ecole Polytechnique - 91128 - Palaiseau Cedex - France
}

\begin{abstract}
SUMMARY
A method for determining fractured rock mass properties is presented here on the basis of homogenisation approach. The rock mass is considered to be a heterogeneous medium composed of intact rock and of fractures. Its constitutive model is studied numerically using Finite Element Method and assimilating the fractures to joint elements (Coste ${ }^{1}$ ). The method has been applied to a granite formation in France. Geological data on different families of fractures have been used for the statistical representation of the fractures. A mesh-generating tool for the medium with high density of fractures has been developed. The mechanical behaviour of the rock mass (elasticity, ultimate strength and hardening law) has been determined assuming linear elasticity and Mohr-Coulomb strength criterion both for the intact rock and the fractures. Evolution of the mechanical strength in different directions has been determined as a function of the mean stress, thanks to various numerical simulations. The mechanical strength appears to be anisotropic due to the preferential orientation of the fractures. The numerical results allowed us to determine an oriented strength criterion for the homogenized rock mass. A 2D constitutive law for the homogenized medium has been deduced from numerical data. A 3D extension of this model is also presented.
\end{abstract}

Keywords : rock mass, fracture, strength criterion, homogenization, elastoplasticity

\section{INTRODUCTION}

Mechanical behaviour a of fractured rock mass depends on the properties of the intact rock and the fractures. The properties of the intact rock are determined by laboratory experiments. These experiments overestimate the strength of the rock mass at large scale whereas its deformability is underestimated. Rock mass classification methods provide empirical estimations for mechanical properties of fractured rock masses (Barton et al. ${ }^{2}$, Bieniawski ${ }^{3}$ ). Semi analytical calculations may provide exact solutions in some special case, for instance when the fractures are parallel to one or two different directions (Bekaert and Maghous ${ }^{4}$, de Buhan and Maghous $^{5}$ ). For the study of underground facilities in a rock mass with a great number of fractures, numerical models based on Discrete Elements Method (DEM) can be used. However, in presence of a very high density of fractures DEM becomes very heavy to handle and requires very long calculations. An example is given farther (§3) for a rock mass in a 
granite located in the West of France. According to geological data, this granite contains a high density of fractures (see Figure 5), mostly filled with recrystalized materials (Calcite), but corresponding to potential discontinuities. One can imagine that due to the very high density of fractures, an attempt to modelling of underground facilities taking account of each fracture may be very heavy, leading to very long calculations. On the other hand, if the dimensions of the underground facilities are great enough compared to the heterogeneity size, a homogenized macroscopic model of the rock becomes easier to handle and more interesting. Many attempts and investigations have been made in this area for materials and rocks (Kachanov $^{6}$, Cai and Horii ${ }^{7,8}$, Le Ravalec and Guéguen ${ }^{9}$, Oda $^{10}$, Budianski and O'Connell ${ }^{11}$, Hashin $^{12}$, Huet ${ }^{13}, \ldots$ ). Most of these attempts have been made for some idealized cases without considering crack interaction. However thus aspect has been taken under account for the study of flow in fractured rocks (Long et al. ${ }^{14}$, Long and Withespoon ${ }^{15}$, Billeux et al. ${ }^{16}$ ). More recently homogenization of fractured rocks has been done using Finite Element numerical models. Coste ${ }^{1}$ has used such a technique for the study of the homogenized hydromechanical behaviour of rock masses using the non linear elastic model of Goodman ${ }^{17}$ for the fractures.

In the present paper, the numerical Finite Element Method has been improved by using an averaging lemma allowing to deduce the homogenized volume quantities from boundary values of nodal forces and displacements. An automatic procedure for mesh generation is also presented for a fractured medium with high density of fractures. Then, the method is applied to the determination of the mechanical properties of a fractured rock mass. The originality of this work is the extension of the previous approach beyond elastic domain, since not only homogenized elastic properties but also mechanical strength, its evolution under bulk stress and as a function of rock deformation (so-called hardening) are determined numerically.

\subsection{General scheme}

\section{NUMERICAL METHOD}

The rock mass is considered as a heterogeneous medium constituted by intact rock and different families of fractures with a statistical distribution of size and orientation and with a given mechanical behaviour. The homogenized strain $\varepsilon^{\text {hom }}$ and stress $\sigma^{\text {hom }}$ of this medium are defined as the average values of the local strain and stress distributions $\varepsilon$ and $\sigma$ in the medium (Figure 1). The homogenized behaviour of this medium, i.e., the relationships between $\varepsilon^{\text {hom }}$ and $\sigma^{\text {hom }}$, are determined by numerical calculations. For this purpose, displacements or tractions corresponding to homogeneous macroscopic strains or stresses are prescribed on the boundary of a sufficiently large domain (Representative Elementary Volume, REV). Strains and stresses in this domain are calculated using Finite Element Method and representing the fractures by joint elements $\left(\right.$ Coste $\left.^{1}\right)$. The average values of strains and stresses in the domain are then calculated. The REV size is determined by a preliminary investigation. For this purpose, values of elastic parameters, especially those of the Young modulus, are calculated on domains of increasing sizes. The REV size corresponds to the minimum size beyond which the values obtained for elastic parameters are stable (not fluctuating). In such a condition, the average values of displacements and strains do not show any significant fluctuation under a constant 
stress (or force) applied on the boundary. Also, the average stress does not change significantly when the boundaries are submitted to constant displacements.

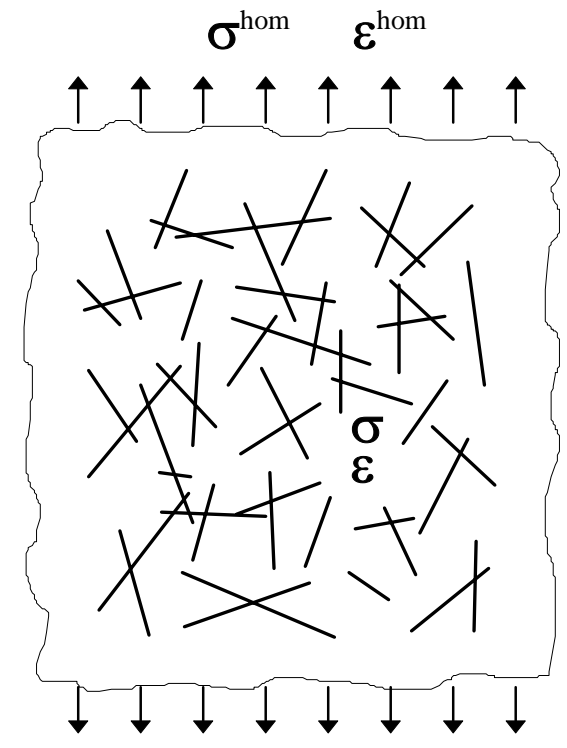

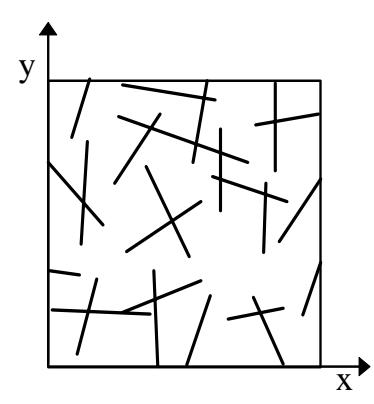

Representative Elementary Volume

Figure 1. The fractured rock mass considered as an heterogeneous medium constituted of the intact rock and fractures

\subsection{Mesh generation}

One major difficulty is the mesh generation for Finite Element computation in presence of a high density of fractures. The elements must not contain any fracture inside them; the fractures have to be located only on the boundary of the elements in order to be assimilated to joint elements. A numerical tool was developed able to generate such a mesh automatically. The automatic procedure for mesh generation is the following : the domain containing fractures is first divided in a number of closed sub-domains by introducing additional lines in the domain which connect the end of the fractures but do not intersect with the existing fractures or lines (figure 2). These closed sub-domains are then discretized individually using classical methods. The meshes corresponding to the individual domains are finally assembled to form the mesh for the whole domain. The interface between different sub-domains is modelled by joint elements only if the interface corresponds to a fracture.

Indeed, the division of the whole domain in a set of sub-domains can be made in different ways, therefore, the mesh generation solution is not unique. But, as it is the case of any other mechanical problem studied by FEM, it can be shown that when the mesh is sufficiently fine, the final result does not depend upon the mesh. 


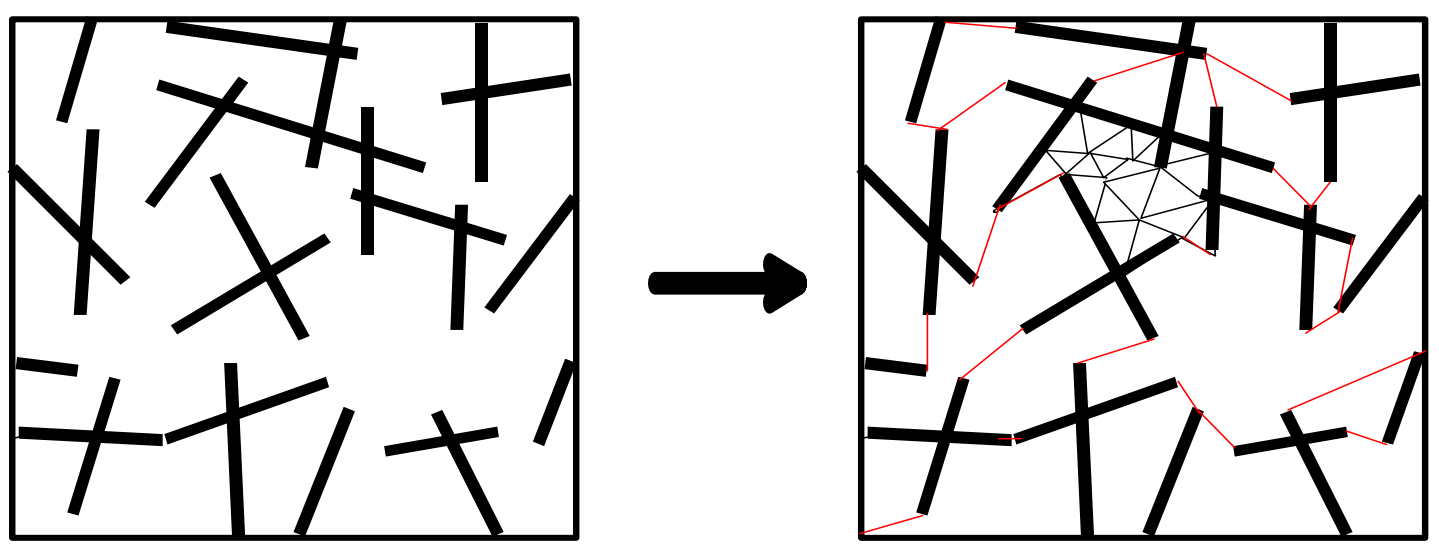

Figure 2 : Method of mesh making for the fractured domain by an automatic procedure : subdivision of the domain in closed sub-domains not containing fractures, discretizing subdomains individually and assembling the individual meshes to form the whole mesh.

\subsection{Averaging method}

The transition between "microscopic" and "macroscopic" variables requires averaging strain and stress fields over the whole domain. For this purpose, the following basic lemma (Hill ${ }^{18,19}$, François et al. ${ }^{20}$ ) is used :

Let the straightforward volume averages of Cartesian components of Cauchy stress $\sigma$ and infinitesimal strain $\varepsilon$ be denoted by $\bar{\sigma}$ and $\bar{\varepsilon}$. In relation to a given domain, the surface tractions or displacements that would be generated by uniform internal fields $\bar{\sigma}$ or $\bar{\varepsilon}$ will be spoken of as uniform loading or uniform constraint respectively.

Then, let $\sigma$ be any bounded and self equilibrated field of stress and $\varepsilon$ any field of infinitesimal strain derivable from a continuous displacement. Then, when the boundary conditions for one or the other are uniform, the volume average of their scalar product can be precisely evaluated as the scalar product of their volume average $\left(\mathrm{Hill}^{18}\right)$ :

$$
\overline{\sigma: \varepsilon}=\bar{\sigma}: \bar{\varepsilon}
$$

Uniform displacement boundary conditions on the boundary $\partial \mathrm{V}$ of a volume $\mathrm{V}$ are defined by :

$$
\forall \mathbf{x} \in \partial \mathrm{V}, \quad \mathrm{u}_{\mathrm{i}}(\mathbf{x})=\mathrm{B}_{\mathrm{ij}} \mathrm{x}_{\mathrm{j}}
$$

where B is a constant symmetric tensor. Consider now a displacement field u corresponding to the uniform boundary conditions (2) and the strain field $\varepsilon$ deriving from $\mathrm{u}: \varepsilon_{\mathrm{ij}}=\left(\partial_{\mathrm{i}} \mathrm{u}_{\mathrm{j}}+\partial_{\mathrm{j}} \mathrm{u}_{\mathrm{i}}\right) / 2$. Integration of $\partial_{\mathrm{i}} \mathrm{u}_{\mathrm{j}}$ over $\mathrm{V}$ gives :

$$
\int_{V} \partial_{i} u_{j} d v=\int_{\partial V} u_{j} n_{i} d s=\int_{\partial V} B_{i k} x_{k} n_{i} d s=B_{i k} \int_{\partial V} x_{k} n_{i} d s=V B_{i j}
$$

where the following identity has been used : 
This allows to show that :

$$
\int_{\partial \mathrm{V}} \mathrm{x}_{\mathrm{k}} \mathrm{n}_{\mathrm{i}} \mathrm{ds}=\int_{\mathrm{V}} \partial_{\mathrm{i}} \mathrm{x}_{\mathrm{k}} \mathrm{dv}=\mathrm{V} \delta_{\mathrm{kj}}
$$

$$
\bar{\varepsilon}=\left(B+B^{T}\right) / 2
$$

Uniform traction boundary conditions on $\partial \mathrm{V}$ are defined by a surface traction density $\mathrm{T}$ on $\partial \mathrm{V}$ given by :

$$
\forall \mathbf{x} \in \partial \mathrm{V}, \quad \mathrm{T}_{\mathrm{i}}(\mathbf{x})=\mathrm{C}_{\mathrm{ij}} \mathrm{n}_{\mathrm{j}}(\mathbf{x})
$$

Consider a self-equilibrated stress field $\sigma$ corresponding to the uniform boundary conditions (4). Writing :

$$
\int_{V} \sigma_{i j} d v=\int_{V} \sigma_{i k} \delta_{k j} d v=\int_{V} \sigma_{i k} \partial_{k} x_{j} d v=\int_{\partial V} \sigma_{i k} x_{j} n_{k} d s-\int_{V}\left(\partial_{k} \sigma_{i k}\right) x_{j} d v
$$

and taking account of the equilibrium equation in $\mathrm{V}, \partial_{\mathrm{k}} \sigma_{\mathrm{ik}}=0$, the boundary condition, $\sigma_{\mathrm{ik}} \mathrm{n}_{\mathrm{k}}$ $=\mathrm{C}_{\mathrm{ik}} \mathrm{n}_{\mathrm{k}}$ on $\partial \mathrm{V}$, and of (3), one finds :

$$
\bar{\sigma}=\mathrm{C}
$$

Consider now a domain $\mathrm{V}$ and a loading on $\partial \mathrm{V}$ consisting in a surface traction density $\mathrm{T}$ or in displacement increments $\delta U$. Furthermore, let $\sigma$ and $\delta \varepsilon$ denote the stress and strain fields resulting from this loading in $\mathrm{V}$. The virtual work principle allows to express :

$$
\delta \mathrm{W}_{\mathrm{e}}=\delta \mathrm{W}_{\mathrm{i}} \Rightarrow \int_{\partial \mathrm{V}} \mathrm{T} . \delta \overrightarrow{\mathrm{U}} \mathrm{ds}=\int_{\mathrm{V}} \sigma: \delta \varepsilon \mathrm{dv}
$$

Assuming that the prescribed boundary conditions $\mathrm{T}$ or $\delta \mathrm{U}$ are uniform, the above lemma allows to write :

$$
\int_{\mathrm{V}} \sigma: \delta \varepsilon \mathrm{dv}=\mathrm{V} \overline{\sigma: \delta \varepsilon}=\mathrm{V} \bar{\sigma}: \delta \bar{\varepsilon}
$$

and so the following equality is derived :

$$
\frac{1}{\mathrm{~V}} \int_{\partial \mathrm{V}} \mathrm{T} \cdot \delta \mathrm{U} \mathrm{ds}=\bar{\sigma}: \delta \bar{\varepsilon}
$$

If uniform displacement boundary conditions are prescribed then $\delta U$ and $\delta \bar{\varepsilon}$ are known, and (6) allows to calculate $\bar{\sigma}$ as a function of the boundary tractions T. In the same way, if traction conditions are prescribed, (6) allows to calculate $\delta \bar{\varepsilon}$ as a function of the boundary displacement $\delta U$. It can be showed that the above equality holds for combined force and displacement boundary conditions, i.e., when the boundary conditions correspond to $\mathrm{u}_{\mathrm{i}}(\mathbf{x})=\mathrm{B}_{\mathrm{ij}} \mathrm{x}_{\mathrm{j}}$ on a part of $\partial \mathrm{V}$ and to $\mathrm{T}_{\mathrm{i}}(\mathbf{x})=\mathrm{C}_{\mathrm{ij}} \mathrm{n}_{\mathrm{j}}(\mathbf{x})$ on the complementary part.

\subsection{Numerical experiments}

Let us assume that the considered domain is a rectangle with side $L_{x}$ in $x$ direction and $L_{y}$ in $\mathrm{y}$ direction, and let us number the sides from 1 to 4 as shown in Figure 3. 


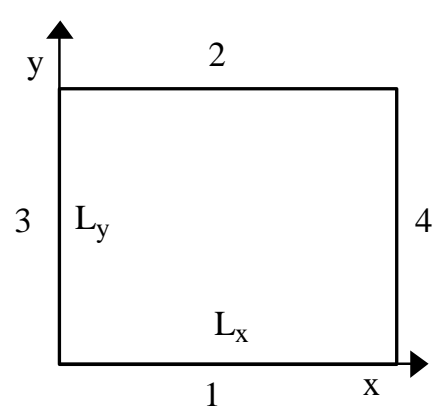

Figure 3. Sides numbers of the sides of the VER rectangle

As well, let the following loading cases be considered :

a) Compression in $\mathrm{x}$ direction with different values of lateral stress $\sigma_{\mathrm{yy}}$ :

A constant displacement $\delta \mathrm{U}_{\mathrm{y}}$ is prescribed on the side (1) and (2) with $\delta \mathrm{U}_{\mathrm{y}}(1)=0$, and a constant surface traction $T_{\mathrm{x}}$ is prescribed on the sides 3 and 4 , with $T_{\mathrm{x}}(4)=-\mathrm{T}_{\mathrm{x}}(3)$.

b) Compression in y direction with different values of lateral stress $\sigma_{\mathrm{xx}}$ :

A constant displacement $\delta \mathrm{U}_{\mathrm{x}}$ is prescribed on the sides (3) and (4) with $\delta \mathrm{U}_{\mathrm{x}}(3)=0$, and a constant surface traction $\mathrm{T}_{\mathrm{y}}$ is prescribed on the sides 1 and 2 , with $\mathrm{T}_{\mathrm{y}}(2)=-\mathrm{T}_{\mathrm{y}}(1)$.

c) Shear in xy direction with different values of the mean stress $\sigma_{\mathrm{m}}=\sigma_{\mathrm{xx}}=\sigma_{\mathrm{yy}}$ :

A constant displacement $\delta U_{x}$ is prescribed on the sides (1) and (2), and a constant displacement $\delta \mathrm{U}_{\mathrm{y}}$ is prescribed on the sides (3) and (4) with $\delta \mathrm{U}_{\mathrm{x}}(1)=\delta \mathrm{U}_{\mathrm{y}}(3)=0$, and $\delta \mathrm{U}_{\mathrm{x}}(2)=$ $\delta \mathrm{U}_{\mathrm{y}}(4)$, and a constant normal surface traction $\mathrm{T}$ is prescribed on all the sides $\left(\mathrm{T}=\mathrm{T}_{\mathrm{y}}(1)=\right.$ $\left.\mathrm{T}_{\mathrm{y}}(2)=-\mathrm{T}_{\mathrm{x}}(4)=\mathrm{T}_{\mathrm{x}}(3)\right)$.

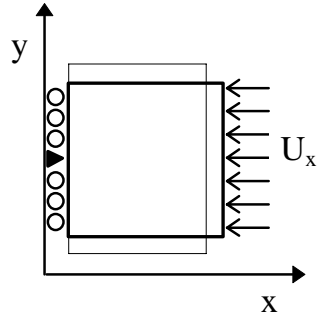

(a)

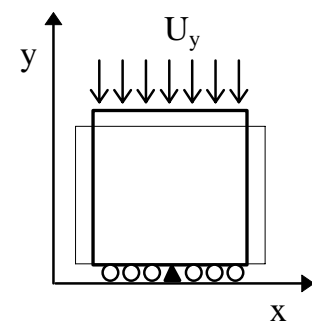

(b)

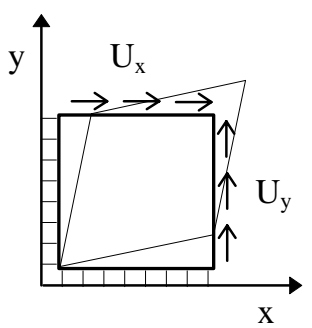

(c)

Figure 4. Numerical experiments :

(a) Compression in $\mathrm{x}$ direction, (b) in y direction, (c) shear in xy direction

These loadings are special cases of combined uniform loadings, the particularity of which is that the prescribed displacement or surface traction is constant on each side of the domain. In these cases, the calculation of the first member of (6) and so the determination of the homogenized quantities are very easy. In fact let $\delta \overline{\mathrm{u}}(\mathrm{n})$ designate the average displacement on the nodes of the side number $n(n=1, \ldots, 4)$, and by $\vec{F}(n)$ the total force on the nodes of the side number $n(n=1, \ldots, 4)$. Following different loading cases, these quantities are deduced either from the prescribed conditions or from the calculation results. The equation (6) and the fact that boundary conditions are constant along each side, allow to obtain different components of $\delta \bar{\varepsilon}$ and of $\bar{\sigma}$ by means of the following formulas : 


$$
\begin{aligned}
& \delta \bar{\varepsilon}_{\mathrm{xx}}=\left[\delta \overline{\mathrm{u}}_{\mathrm{x}}(4)-\delta \overline{\mathrm{u}}_{\mathrm{x}}(3)\right] / \mathrm{L}_{\mathrm{x}}, \quad \bar{\varepsilon}_{\mathrm{yy}}=\left[\delta \overline{\mathrm{u}}_{\mathrm{y}}(2)-\delta \overline{\mathrm{u}}_{\mathrm{y}}(1)\right] / \mathrm{L}_{\mathrm{y}}, \\
& \delta \bar{\varepsilon}_{\mathrm{xy}}=\frac{1}{2}\left\{\left[\delta \overline{\mathrm{u}}_{\mathrm{x}}(2)-\delta \overline{\mathrm{u}}_{\mathrm{x}}(1)\right] / \mathrm{L}_{\mathrm{y}}+\left[\delta \overline{\mathrm{u}}_{\mathrm{y}}(4)-\delta \overline{\mathrm{u}}_{\mathrm{y}}(3)\right] / \mathrm{L}_{\mathrm{x}}\right\} \\
& \bar{\sigma}_{\mathrm{xx}}=\frac{1}{2}\left[\overrightarrow{\mathrm{F}}_{\mathrm{x}}(4)-\overrightarrow{\mathrm{F}}_{\mathrm{x}}(3)\right] / \mathrm{L}_{\mathrm{y}}, \quad \bar{\sigma}_{\mathrm{yy}}=\frac{1}{2}\left[\overrightarrow{\mathrm{F}}_{\mathrm{y}}(2)-\overrightarrow{\mathrm{F}}_{\mathrm{y}}(1)\right] / \mathrm{L}_{\mathrm{x}} \\
& \bar{\sigma}_{\mathrm{xy}}=\frac{1}{2}\left\{\left[\overrightarrow{\mathrm{F}}_{\mathrm{x}}(2)-\overrightarrow{\mathrm{F}}_{\mathrm{x}}(1)\right] / \mathrm{L}_{\mathrm{x}}+\left[\overrightarrow{\mathrm{F}}_{\mathrm{y}}(4)-\overrightarrow{\mathrm{F}}_{\mathrm{y}}(3)\right] / \mathrm{L}_{\mathrm{y}}\right\}
\end{aligned}
$$

One can also notice that equilibrium conditions imply the following equalities :

$$
\sum_{n=1,4} \vec{F}_{x}(n)=\sum_{n=1,4} \vec{F}_{y}(n)=0
$$

The homogenized stress and strain are calculated for each load case using the method presented above. Strain-stress curves can be calculated in this way for different loading paths (see Figure 8). The constitutive model for the material (homogenized medium) can be determined through these curves.

\subsection{Fracture distribution}

\section{FRACTURED ROCK MASS}

A 2D representation of a granitic fractured rock mass situated in the West of France is considered here. The $\mathrm{x}$ axis in this representation corresponds to the horizontal North-South direction, and the y axis, to the vertical direction. The fractures size, orientation, density and aperture or thickness (filled fractures) introduced in this model have been deduced from the geological data. The possible propagation of existing fractures that may locally occur is not considered. This question is of second order regarding the intensity of existing fractures and also the insufficient data available specially on the extension of fractures. The extension of the existing fractures varies from $20 \mathrm{~cm}$ to $30 \mathrm{~m}$ and their slope (angle with the $\mathrm{x}$ axis) varies from 0 to $90^{\circ}$. The statistical distribution of the fractures extension is assumed to obey to an exponential law with an average value of $10 \mathrm{~m}$. The orientation obeys to a normal distribution law with an average value of $60^{\circ}$ (sub-vertical fractures) and a $10^{\circ}$ variance. The fractures density is uniform and equal to $0.4 \mathrm{~m}^{-2}$. The value of the fractures thickness, required for determination of the joint behaviour, is assumed to be about a few millimetres on the basis of some core samples observation. A statistical representation of these fractures is given in Figure 5 for a square domain with $50 \mathrm{~m}$ side. 


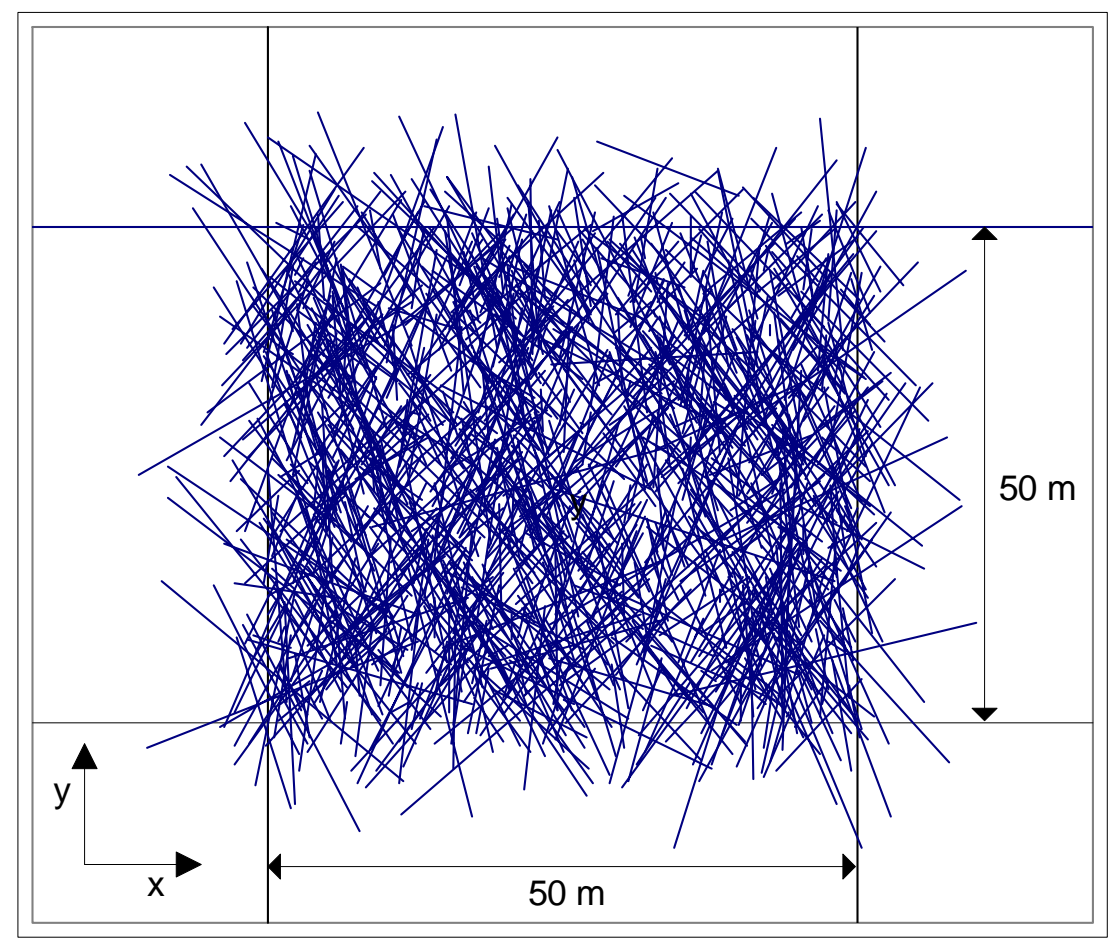

Figure 5. Fracture distribution for a fractured rock mass deduced from geological data

\subsection{Constitutive models of intact rock and fractures}

According to the results of laboratory tests on the rock without any fracture and on single fractures, the intact rock has a linear and isotropic behaviour in the elastic stage, with values of Young modulus $\mathrm{E}=72000 \mathrm{MPa}$ and Poisson ratio $v=0.25$. The strength properties of the intact rock are given by a Mohr-Coulomb criterion with cohesion $\mathrm{C}=17 \mathrm{MPa}$ and internal friction angle $\phi=57^{\circ}$. The fractures behaviour in the elastic domain is assumed to be linear and characterized by normal and tangential stiffness $k_{n}$ and $k_{t}$ respectively. Beyond the elastic domain, Mohr-Coulomb criterion is used with cohesion $\mathrm{c}=1.51 \mathrm{MPa}$ and internal friction angle $\varphi=27^{\circ}$. These simple models (linear elasticity and Mohr-Coulomb criterion) allows to compare easily the homogenized rock mass behaviour with the behaviour of its constituents (intact rock and fractures). More representative models of fracture behaviour (non linear elasticity) can easily be taken into account if needed. The values of fractures parameters considered here have been obtained by laboratory measurements on some natural fractures with a few millimetres of thickness and filled with recrystallized calcite. Because of the high value of the intact rock cohesion compared to the fractures cohesion $(\mathrm{C}=17 \mathrm{MPa}, \mathrm{c}=1.51 \mathrm{MPa})$, the fractures reach their elastic limit before the intact rock. Therefore, the damage behaviour of the rock mass is largely determined by the fractures parameters, and in the loading paths presented in the following, The intact rock essentially remains elastic. In order to simplify the calculation, the elastic-brittle behaviours of the intact rock and the fractures are replaced by an elastic-perfectly plastic behaviour. For monotonous increasing loading paths studied here, 
when the unloading does not take place, this simplification does not change significantly the macroscopic results.

\section{NUMERICAL STRESS-STRAIN CURVES}

A preliminary investigation of the REV size for the considered fractured rock mass, by the method described above ( $\$ 2.1$ ), led to a side value of about $8 \mathrm{~m}$. A square domain of $8 \mathrm{~m}$ side of was submitted to compressive or shear loading paths described in §2.4. The REV and the mesh generated for this REV are presented in Figures 6 and 7 respectively.

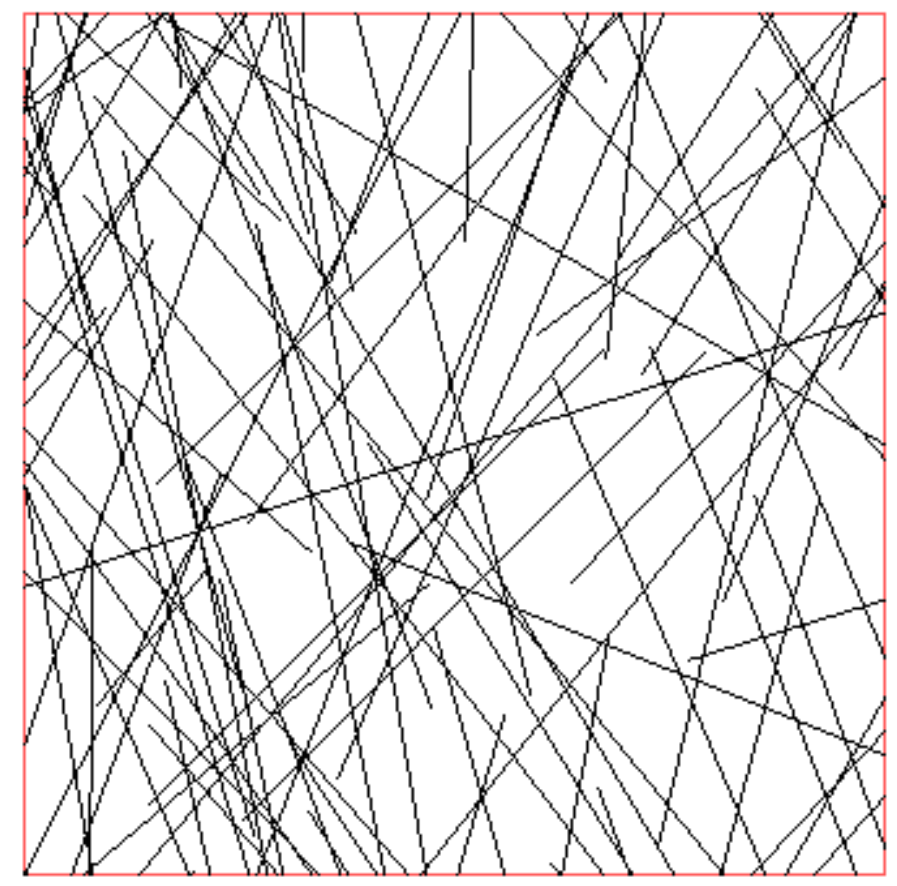

Figure 6 : REV corresponds to a square domain of $8 \mathrm{~m}$ side

Homogenized stresses and strains were calculated using the averaging method presented above. The calculations were made under plane stress assumption. This assumption makes the determination of the strength properties easier since this assumption leads to a stress tensor with only three independent components.

To simplify the notations, the homogenized stresses and strains are denoted by $\sigma$ and $\varepsilon$ in what follows. Stress-strain plots obtained for different loading cases are given in Figure 8. 


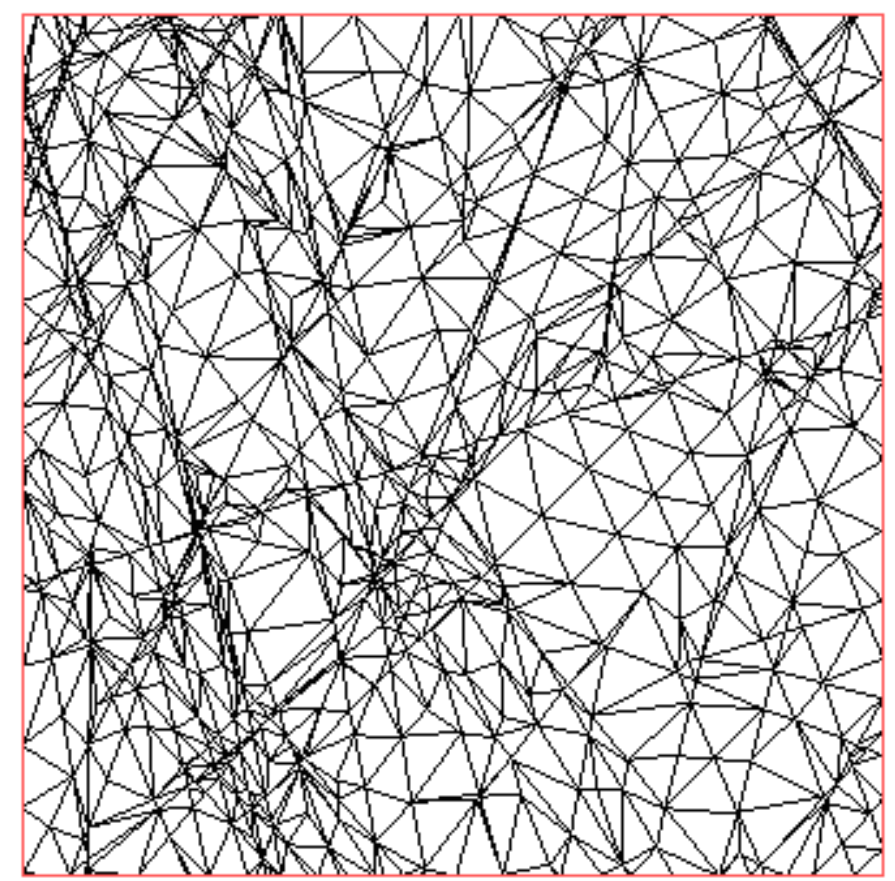

Figure 7 : Discretization of the REV

\section{CONSTITUTIVE MODELLING}

The stress-strain plots given in Figure 8 provide somehow the "experimental data" on the basis of which the constitutive model of the rock mass is to be determined. These data are to be completed, as it is the case of real experimental data, by further assumptions concerning the expression of the constitutive model.

The analysis of the results shows up three different stages of deformation. The stress-strain plots begin with a linear elastic stage. During the following stage, the slopes of the curve decrease but the stress is always increasing (positive hardening stage). The curves then tend to a horizontal asymptote corresponding to a maximum value of the stress which cannot be exceeded (asymptotic perfect plastic behaviour). In the following, first the results corresponding to the elastic stage, then those characterizing the ultimate plastic behaviour, and finally the results corresponding to the transient hardening stage will be analysed. 


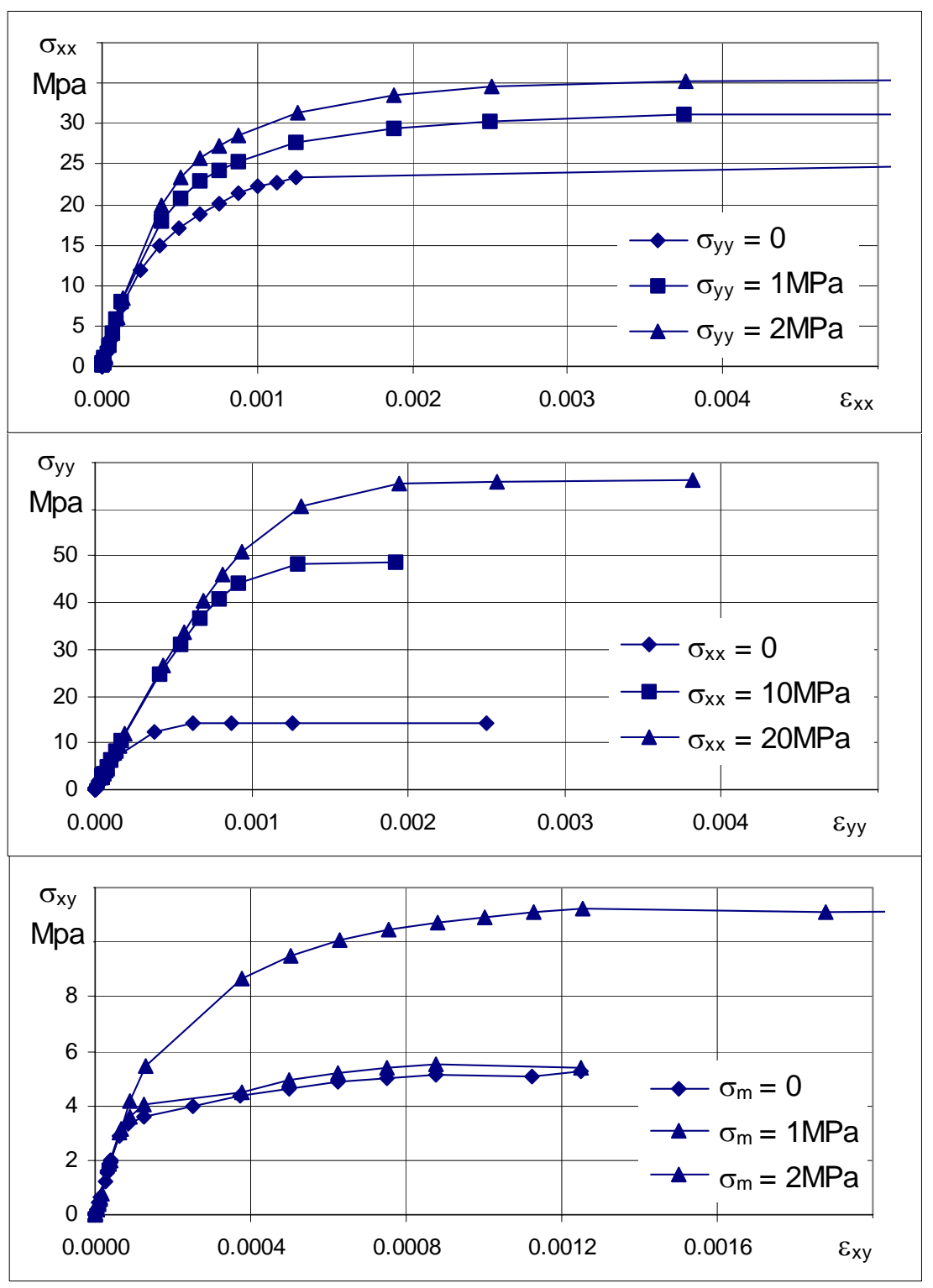

Figure 8. Stress-strain curves for a homogenized fractured rock mass corresponding to different loading paths: compression in $\mathrm{x}$ direction under different values of lateral stress $\sigma_{\mathrm{yy}}$, compression in y direction under different values of lateral stress $\sigma_{\mathrm{xx}}$, and shear in xy direction under different values of the mean stress $\sigma_{\mathrm{m}}=\sigma_{\mathrm{xx}}=\sigma_{\mathrm{yy}}$ (see Figure 4 for the loading paths).

\subsection{Elastic stage}

During the elastic stage, stress-strain curves have a linear trend. This stage extends up to the strain values of about $10^{-5}$. The ratios obtained for different variables during this stage are given in Table 1. This Table shows that when a compressive load is applied in $\mathrm{x}$ or $\mathrm{y}$ direction, very small values are obtained for $\varepsilon_{\mathrm{xy}} / \varepsilon_{\mathrm{xx}}$ or $\varepsilon_{\mathrm{xy}} / \varepsilon_{\mathrm{yy}}$ compared to $\varepsilon_{\mathrm{yy}} / \varepsilon_{\mathrm{xx}}$ or $\varepsilon_{\mathrm{xx}} / \varepsilon_{\mathrm{yy}}$. The shear strain $\varepsilon_{\mathrm{xy}}$ is therefore negligible in these cases. Moreover, the elastic moduli in $\mathrm{x}$ 
and y directions have very close values. For these reasons, the elastic behaviour can be assumed to be isotropic with a good approximation. The average values of Young modulus and Poisson are (Table 1) :

$$
\begin{array}{ll}
\text { Young modulus: } & \mathrm{E}=62.6 \mathrm{GPa} \\
\text { Poisson ratio : } & v=0.267
\end{array}
$$

These values lead to the shear modulus value of $G=E / 2(1+v)=24.7 \mathrm{GPa}$. This value corresponds very accurately to that obtained numerically through a shear loading path (see the last line of Table $1: G=\sigma_{x y} / 2 \varepsilon_{x y}=24.7 \mathrm{GPa}$ ). This result confirms the validity of the hypothesis of linear and isotropic elastic behaviour.

Table 1 : Ratios obtained during the elastic stage

\begin{tabular}{|c|c|c|c|}
\hline Load direction $\mathrm{xx}$ & $\sigma_{\mathrm{xx}} / \varepsilon_{\mathrm{xx}}(\mathrm{GPa})$ & $\varepsilon_{\mathrm{yy}} / \varepsilon_{\mathrm{xx}}$ & $\varepsilon_{\mathrm{xy}} / \varepsilon_{\mathrm{xx}}$ \\
\hline & 63.2 & 0.269 & 0.015 \\
\hline Load direction yy & $\sigma_{\mathrm{yy}} / \varepsilon_{\mathrm{yy}}(\mathrm{GPa})$ & $\varepsilon_{\mathrm{xx}} / \varepsilon_{\mathrm{yy}}$ & $\varepsilon_{\mathrm{xy}} / \varepsilon_{\mathrm{yy}}$ \\
\hline & 62.0 & 0.264 & 0.005 \\
\hline Load direction $\mathrm{xy}$ & $\sigma_{\mathrm{xy}} / \varepsilon_{\mathrm{xy}}(\mathrm{GPa})$ & $\varepsilon_{\mathrm{xx}} / \varepsilon_{\mathrm{xy}}$ & $\varepsilon_{\mathrm{yy}} / \varepsilon_{\mathrm{xy}}$ \\
\hline & 49.4 & -0.014 & -0.03 \\
\hline
\end{tabular}

\subsection{Elastic limit}

Determination of the initial elastic limit is important because it characterizes the initiation of damage which has a big effect on the permeability of the rock mass (important parameter of the disposal safety). The initial elastic limit in different directions can be deduced from the numerical plots. It can also be determined by theoretical calculation based on some simplifying assumptions.

The overall elastic limit corresponds to that of macroscopic (homogenized) stress for under which one of the constituents of the heterogeneous medium reaches its elastic limit. As mentioned above, since the cohesion value of the intact rock is greater than that of the fractures $(\mathrm{C}=17 \mathrm{MPa}, \mathrm{c}=1.51 \mathrm{MPa})$, the fractures reach their elastic limit prior to the intact rock. Let us consider a fracture with an angle $\theta$ with respect to the $\mathrm{x}$ direction. Normal and tangent unit vectors are designated respectively by $\vec{n}=(-\sin \theta, \cos \theta)$ and $\vec{t}=(\cos \theta, \sin \theta)$. Assuming that the shear and normal stresses on this fracture are given by the projection of the homogenized stress tensor $\sigma$ on the fracture's plane is equivalent to neglect the interaction between fractures during the initial elastic stage and to assume that they have infinite size. Normal and shear stresses on the fracture, $\sigma_{\mathrm{n}}$ and $\tau$, are then respectively given by $\sigma_{\mathrm{n}}=\overrightarrow{\mathrm{n}} \cdot \sigma \cdot \overrightarrow{\mathrm{n}}$ and $\tau=\overrightarrow{\mathrm{t}} \cdot \sigma \cdot \overrightarrow{\mathrm{n}}$. The elastic domain for the fracture is given by (compressive stress is supposed as negative) : 


$$
|\tau|<\mathrm{c}-\sigma_{\mathrm{n}} \operatorname{tg} \varphi
$$

This inequality leads to the following condition for the homogenized stress $\sigma$ :

$$
|\overrightarrow{\mathrm{t}} \cdot \sigma \cdot \overrightarrow{\mathrm{n}}|<\mathrm{c}-\overrightarrow{\mathrm{n}} \cdot \sigma \cdot \overrightarrow{\mathrm{n}} \operatorname{tg} \varphi
$$

Considering that under plane stress assumption, the components of the stress tensor are $\sigma_{\mathrm{xx}}$, $\sigma_{\text {yy }}$ and $\sigma_{\mathrm{xy}}$, one can define the mean stress $\sigma_{\mathrm{m}}$ and the deviatoric stress in the plane by the following expressions :

$$
\sigma_{\mathrm{m}}=\frac{1}{2}\left(\sigma_{\mathrm{xx}}+\sigma_{\mathrm{yy}}\right) \quad \mathrm{s}=\frac{1}{2}\left(\sigma_{\mathrm{xx}}-\sigma_{\mathrm{yy}}\right)
$$

Let us consider the domain defined by the inequality (7) in the plane $\left(s, \sigma_{x y}\right)$ for a given value of $\sigma_{\mathrm{m}}$ and let us define in this plane $\overrightarrow{\mathrm{S}}=\left(\mathrm{s}, \sigma_{\mathrm{xy}}\right), \overrightarrow{\mathrm{U}}=(\cos 2 \theta, \sin 2 \theta)$ and $\overrightarrow{\mathrm{V}}=(-\sin 2 \theta$, $\cos 2 \theta)$. The inequality (7) can be written as :

$$
|\vec{S} \cdot \vec{V}|<c-\sigma_{m} \operatorname{tg} \varphi+\vec{S} \cdot \vec{U} \operatorname{tg} \varphi
$$

This condition defines a domain limited by two straight lines $\mathrm{D}_{1}$ and $\mathrm{D}_{2}$ making an angle $\varphi$ with the $\overrightarrow{\mathrm{U}}$ direction (Figure 9). The elastic domain for the set of fractures is the intersection of the domains defined in this way for different values of $\theta$. For the rock mass considered here $\theta$ varies between 0 et $90^{\circ}$, and so, the intersection of the considered domains is a circle centred at the origin $\mathrm{O}$ and with a radius $\mathrm{R}$ given by (see Figure 9) $: \mathrm{R}=|\mathrm{OM}| \sin \varphi$. For the point $\mathrm{M}$, the equation of $\mathrm{D}_{1}$ leads to :

and then :

$$
|\overrightarrow{\mathrm{S}} \cdot \overrightarrow{\mathrm{V}}|=0 \Rightarrow \overrightarrow{\mathrm{S}} \cdot \overrightarrow{\mathrm{U}}=-\mathrm{c} \operatorname{cotg} \varphi+\sigma_{\mathrm{m}} \Rightarrow|\mathrm{OM}|=\mathrm{c} \operatorname{cotg} \varphi-\sigma_{\mathrm{m}}
$$

$$
\mathrm{R}=\mathrm{c} \cos \varphi-\sigma_{\mathrm{m}} \sin \varphi
$$

The elastic domain of the rock mass is the intersection of this domain with the elastic domain of the matrix (intact rock). The latter, defined by the Mohr-Coulomb criterion with cohesion $\mathrm{C}$ and an internal friction angle $\phi$, corresponds, for a given value of $\sigma_{\mathrm{m}}$, to a circle in the plane $\left(\mathrm{s}, \sigma_{\mathrm{xy}}\right)$ centred at $\mathrm{O}$ and with a radius $\mathrm{R}^{\prime}=\mathrm{C} \cos \phi-\sigma_{\mathrm{m}} \sin \phi$. For the values of the parameters c, $\varphi, C$ and $\phi$ given above, the second circle contains always the first one ( $\mathrm{R}^{\prime}>\mathrm{R}$ when $\mathrm{R}>0$ ), and so their intersection is equal to the first one.

In conclusion, the elastic domain of the rock mass in the stress space $\left(\sigma_{\mathrm{m}}, \sigma_{\mathrm{xy}}, \mathrm{s}\right)$ is the circular cone defined by :

$$
\sqrt{\sigma_{\mathrm{xy}}^{2}+\mathrm{s}^{2}}<\mathrm{c} \cos \varphi-\sigma_{\mathrm{m}} \sin \varphi
$$




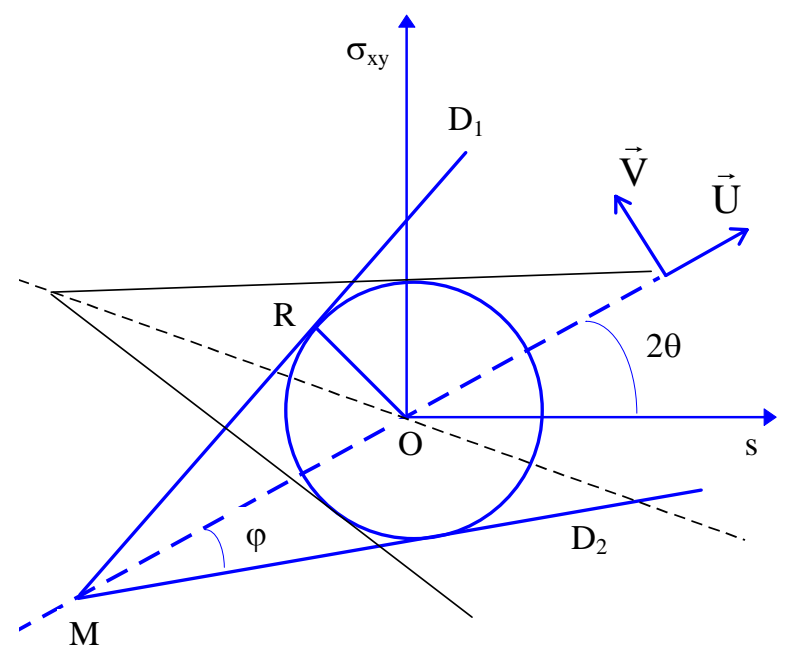

Figure 9 : Elastic domain of the homogenized rock mass

\subsection{Asymptotic stage - strength criterion}

As shown in Figure 8, stress-strain curves tend to horizontal asymptotes. The stress value corresponding to this asymptote represents the ultimate strength of the rock mass in the corresponding direction. This ultimate strength depends on the lateral stresses for compressive loads or on the mean stress for shear loads. The results show up a significant anisotropy related to the plastic behaviour of the rock mass. The strength in $\mathrm{x}$ direction is in fact about 2 times greater than that of $y$ direction. The ultimate stress versus lateral or mean stress has been plotted in Figure 10. This figure shows that the ultimate strength depends linearly on the mean stress. The equations corresponding to the straight lines are given in Figure 10.

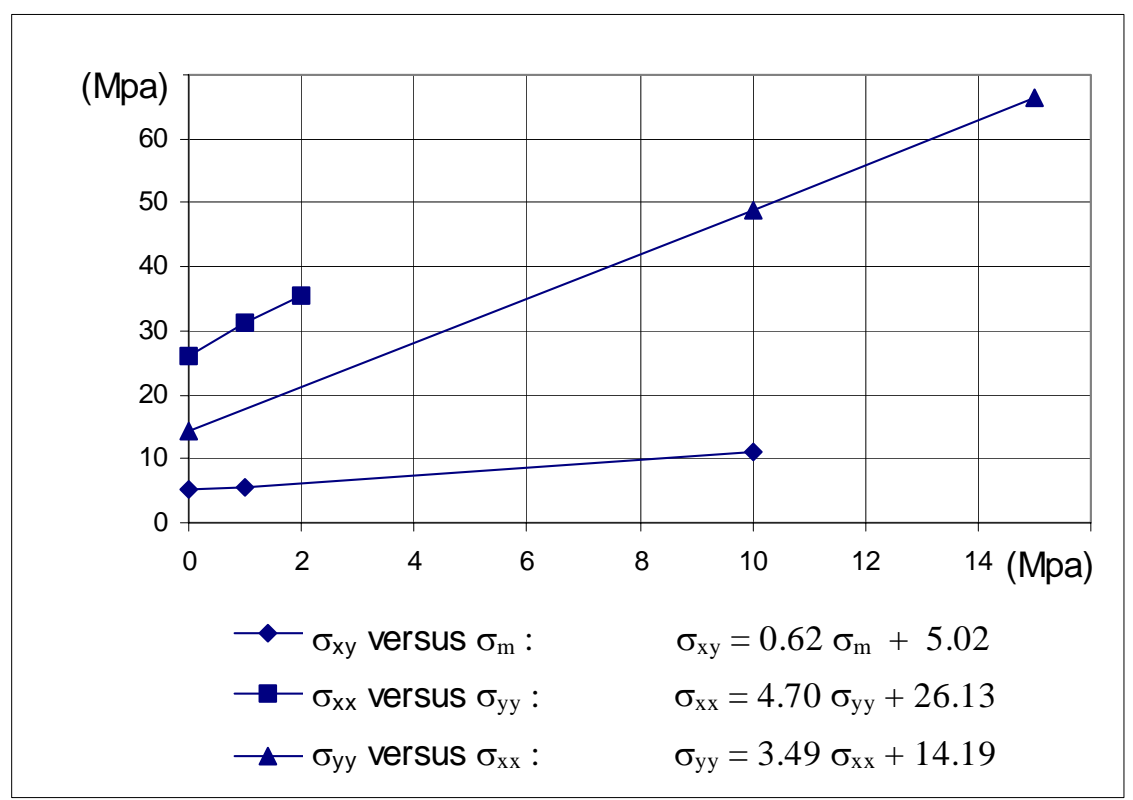

Figure 10 : Effect of lateral or of mean stress on the strength in different directions 
The equation of the line $\sigma_{\mathrm{xy}}$ versus $\sigma_{\mathrm{m}}$ in Figure 10 can be written as : $\sigma_{\mathrm{xy}}=-\sigma_{\mathrm{m}} \operatorname{tg}^{1} \square+\mathrm{C}^{1}$. The two other lines of this figure define linear dependency of $\mathrm{s}$ upon $\sigma_{\mathrm{m}}$ which can be written as $s=-\sigma_{m} \operatorname{tg} \phi^{i}+C^{i}$ with $i=2,3$. The parameters $\phi^{i}$ and $C^{i} \quad(i=1,2,3)$ define the values of the homogenized internal friction angle $\phi^{\text {hom }}$ and cohesion $C^{\text {hom }}$ in different directions. They are given in Table 2 :

Table 2 : Homogenized cohesion and internal friction angle in different directions

\begin{tabular}{|c|r|c|}
\hline Load direction & $\begin{array}{c}\mathrm{C}^{\text {hom }} \\
(\mathrm{MPa})\end{array}$ & $\phi^{\text {hom }}$ \\
\hline $\mathrm{Xx}$ & 4.58 & $33.0^{\circ}$ \\
\hline $\mathrm{Yy}$ & 3.16 & $29.0^{\circ}$ \\
\hline $\mathrm{Xy}$ & 5.02 & $31.8^{\circ}$ \\
\hline
\end{tabular}

One can notice that the values obtained for homogenized cohesion and internal friction angle are intermediate between those of the intact rock $\left(\mathrm{C}=17 \mathrm{MPa}, \phi=57^{\circ}\right)$ and the fractures $\left(\mathrm{c}=1.51 \mathrm{MPa}, \varphi=27^{\circ}\right)$.

These results can be used to elaborate a model for the ultimate strength of the rock mass. The strength domain in the stress space $\left(\sigma_{\mathrm{m}}, \sigma_{\mathrm{xy}}, \mathrm{s}\right)$ can be described by a yield function $\mathrm{f}$; the strength domain corresponding to domain of stresses verifying $f\left(\sigma_{m}, \sigma_{x y}, s\right) \leq 0$. The function $f$ has to be determined using the numerical results given above in Figure 10. According to these results, the intersection of the boundary of the strength domain, corresponding to the surface $\mathrm{f}\left(\sigma_{\mathrm{m}}, \sigma_{\mathrm{xy}}, \mathrm{s}\right)=0$, and the planes $\sigma_{\mathrm{xy}}=0$ and $\mathrm{s}=0$ in the stress space, gives the straight lines represented in Figure 10. For a given value of $\sigma_{\mathrm{m}}$, these results, represented also by the data given in Table 2 , define 3 points in the plane $\left(\sigma_{\mathrm{xy}}, \mathrm{s}\right)$. For $\sigma_{\mathrm{m}}=0$, these points are respectively $(0,4.58),(0,-3.16)$ and $(5.02,0)$. They can be completed by the point corresponding to the shear strength in -xy direction (shear loading with $\sigma_{x y}<0$, not represented in Figure 8). A numerical simulation led to the value of 2.17 MPa for this strength. The four points obtained in this way have been presented in Figure 11. They can be supposed to form a circle. The position of the centre and the radius of this circle vary with $\sigma_{\mathrm{m}}$.

Furthermore, an expression for the yield criterion $\mathrm{f}$ has to be found verifying these properties :

(a) : the intersections of the surface $f=0$ with the planes $\sigma_{x y}=0$ and $s=0$ define straight lines in these planes,

(b) : the intersection of the surface $\mathrm{f}=0$ with the plane $\sigma_{\mathrm{m}}=0$ defines a circle.

An expression for $\mathrm{f}$ (a simple one which is not a unique one) can be proposed as following :

$$
f\left(\sigma_{m}, \sigma_{x y}, s\right)=\left(\sigma_{x y}-D\right)^{2}+(s-D)^{2}-\left(-A \sigma_{m}+G\right)^{2}-D^{2}
$$

As yield criteria are usually supposed to have the dimension of stress, this expression for $f$ will be changed into the following :

$$
f\left(\sigma_{m}, \sigma_{x y}, s\right)=\sqrt{\left(\sigma_{x y}-D\right)^{2}+(s-D)^{2}}-\sqrt{\left(-A \sigma_{m}+G\right)^{2}+D^{2}}
$$


Indeed, these two expressions define the same domain in the stress space. The parameters $A, D$ and $G$ must be determined using the numerical data. The comparison between the expressions of $\mathrm{f}$ in the cases $\sigma_{\mathrm{xy}}=0$ or $\mathrm{s}=0$ allows to identify $\mathrm{A}=\operatorname{tg} \phi^{\text {hom }}$. The expression given for $\mathrm{f}$ assumes that the internal friction angle have the same value in all the (loading) directions. This provides a satisfactory approximation since $\phi^{\text {hom }}$ has very similar values in different directions (Table 2). So, $\phi^{\text {hom }}$ can be supposed to be constant and equal to the average value of $31.3^{\circ}$, and then $A=\operatorname{tg} 31.3^{\circ}=0.608$. Now, considering the case $\sigma_{\mathrm{m}}=0$, the equation $\mathrm{f}=0$ where $\mathrm{f}$ is given by (11), defines a circle in the plane $\left(\sigma_{\mathrm{xy}}, \mathrm{s}\right)$ centred at the point $(D, D)$ and having a radius $R=\left(G^{2}+D^{2}\right)^{1 / 2}$. The values of the two constants $D$ and $G$ are obtained by fitting the four points known in the plane $\left(\sigma_{x y}, s\right)$ (Figure 11). These constants are calculated by least square minimisation of the distances of the points to the circle. This leads to $: \mathrm{D}=1.07 \mathrm{MPa}$ and $\mathrm{G}=3.73 \mathrm{MPa}$.
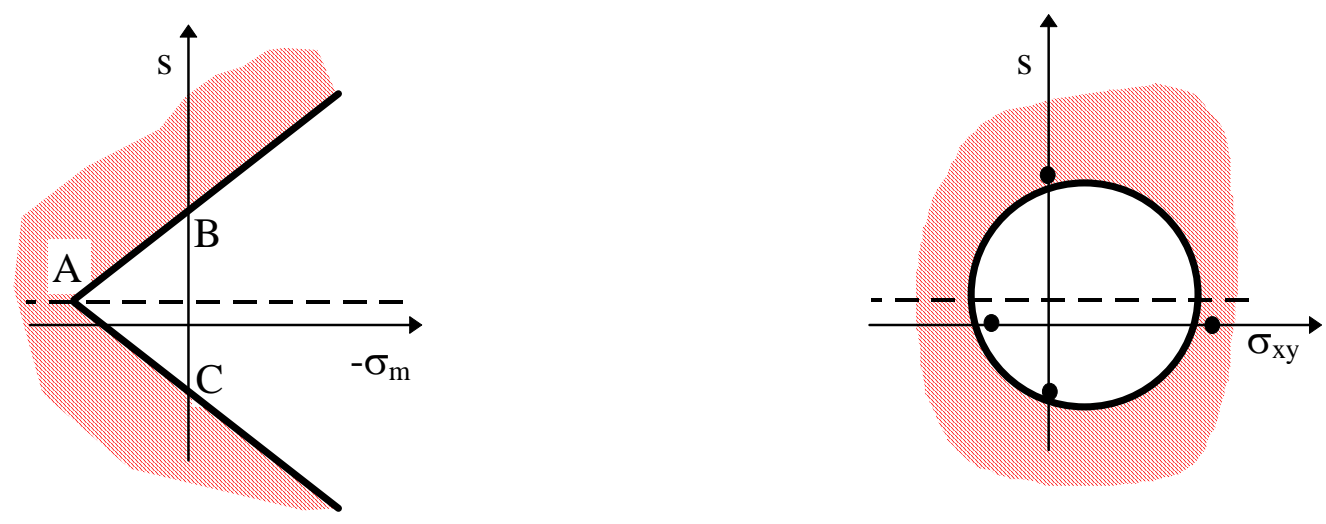

Figure 11. The strength domain in $\left(\sigma_{\mathrm{m}}, \mathrm{s}\right)$ plane is bounded by two straight lines making an angle $\pm 31.3^{\circ}$ with $-\sigma_{\mathrm{m}}$ axis, whereas the strength domain in $\left(\sigma_{\mathrm{xy}}, \mathrm{s}\right)$ plane is approximately a circle.

\subsection{Hardening stage}

The hardening stage begins when the representative stress point reaches the boundary of the initial elastic domain. As seen above, the initial elastic domain is a cone with axis parallel to the $\sigma_{\mathrm{m}}$ axis. For a given value of $\sigma_{\mathrm{m}}$, the elastic domain in the plane $\left(\sigma_{\mathrm{xy}}, \mathrm{s}\right)$ is a circle centred at the origin and with a radius $R=c \cos \varphi-\sigma_{m} \sin \varphi$, where $c$ and $\varphi$ are respectively the cohesion and the internal friction angle of the fractures. This circle is located within the circle corresponding to the ultimate strength (Figure 12). During the hardening stage, the representative stress point follows a path beginning at the boundary of the initial elastic domain and tending to the ultimate strength boundary. This path corresponds to the transient stage described above in the stress-strain plots. 

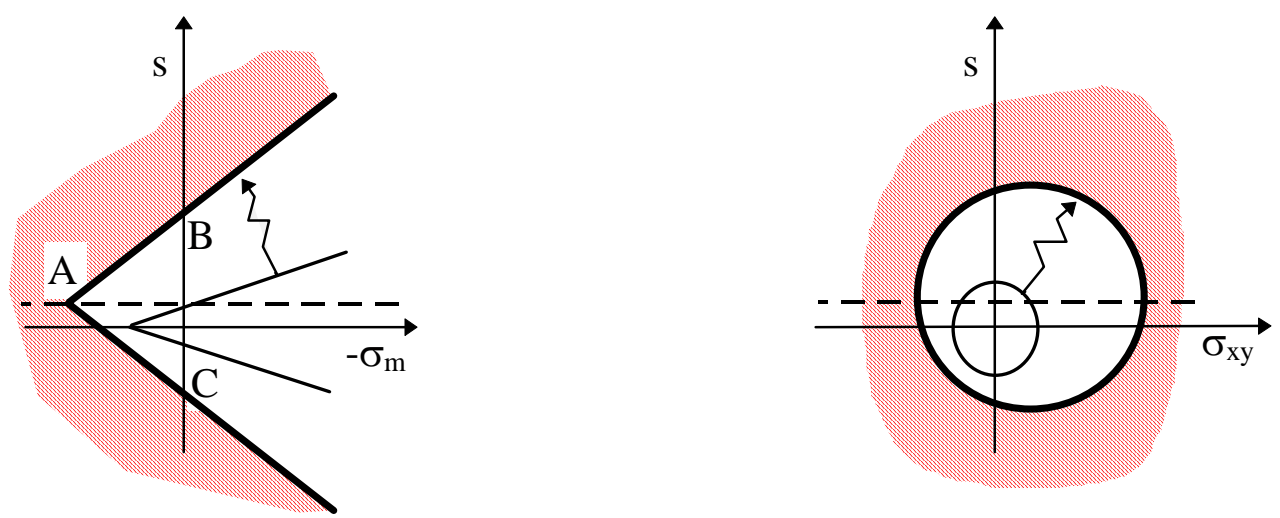

Figure 12. During the hardening stage, the representative stress point follows a path going from the initial elastic boundary to the ultimate strength boundary.

Since the initial elastic boundary given by (10) corresponds to the same expression (11) for adequate values of parameters $\mathrm{A}, \mathrm{D}$ and $\mathrm{G}$, one can assume that the elastic boundary at any stage of deformation can be given by the same expression in which the parameters A,D and G may change. The initial values of these parameters are : $\mathrm{A}_{0}=\sin 27^{\circ}=0.454, \mathrm{D}_{0}=0$ and $\mathrm{G}_{0}=$ $1.51 \cos 27^{\circ}=1.35 \mathrm{MPa}$. The final (or asymptotic limit) values are those obtained above : $\mathrm{A}_{\infty}$ $=0.61, \mathrm{D}_{\infty}=1.07 \mathrm{MPa}$ and $\mathrm{G}_{\infty}=3.73 \mathrm{MPa}$. Regarding the shape of the strain-stress plots, the evolution of these parameters with strain can be assumed to be given by an exponential expression :

$$
A=A_{0}+\left(A_{\infty}-A_{0}\right)\left(1-e^{-\beta \xi}\right)
$$

In this expression, the hardening parameter $\xi$ is defined by $\dot{\xi}=\left\|\dot{\varepsilon}^{\mathrm{p}}\right\|$ with an initial value $\xi=0$, and $\beta$ is a constant parameter which has to be determined by fitting the stress-strain plots in the transient stage. The fitting of different stress-strain plots corresponding to different loading directions, leads to different values of $\beta$ varying between 547 and 2447. This means that $\beta$ depends on the stress orientation. However, this parameter describes the evolution during the transient hardening stage and has no effect on the ultimate strength properties (see Figure 13). Therefore, in case one is interested in the ultimate strength properties of the rock masse, a constant value can be assumed for this parameter in order to simplify the model. A constant value of $\beta=1000$ for all the directions has been considered in the following, and an example of curve fitting using this value of $\beta$ is shown in Figure 13. 


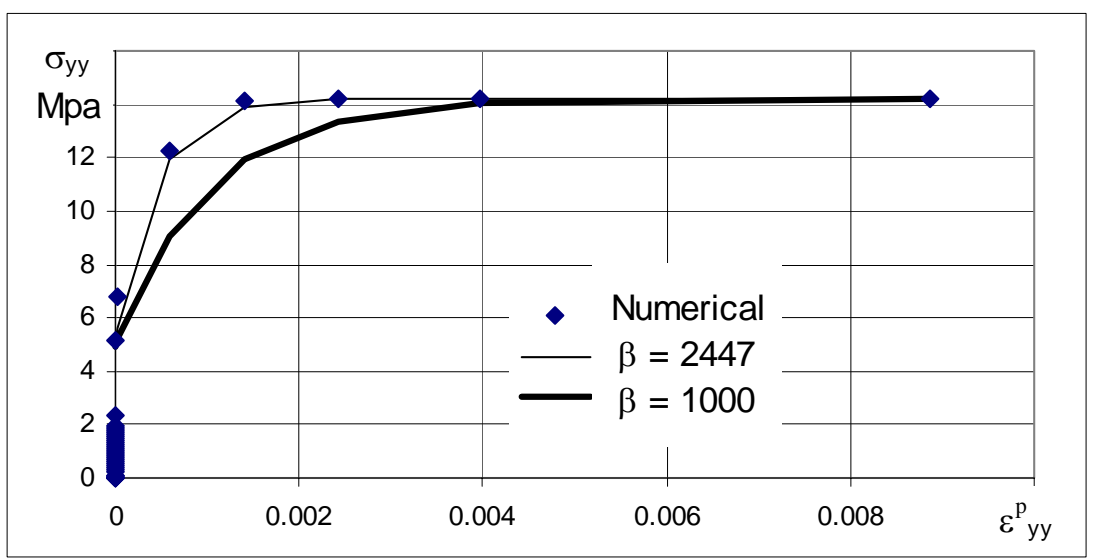

Figure 13. Comparison between the numerical plot (corresponding to compression in $y$ direction), the individual fitting of this plot, leading to $\beta=2447$, and fitting of this plot with a fixed value of $\beta=1000$.

\section{CONSTITUTIVE MODEL}

7.

The homogenized behaviour of the fractured rock is finally defined by the following constitutive model in 2D :

\section{Yield Criterion :}

with :

$$
f(\sigma, \xi)=\leq 0
$$

$$
f(\sigma, \xi): \sqrt{\left(\sigma_{x y}-D\right)^{2}+(s-D)^{2}}-\sqrt{\left(-A \sigma_{m}+G\right)^{2}+D^{2}}
$$

and :

$$
\mathrm{s}=\left(\sigma_{\mathrm{xx}}-\sigma_{\mathrm{yy}}\right) / 2 \quad, \quad \sigma_{\mathrm{m}}=\left(\sigma_{\mathrm{xx}}+\sigma_{\mathrm{yy}}\right) / 2
$$

\section{Hardening law :}

$$
\begin{gathered}
\dot{\mathrm{A}}=\beta\left(\mathrm{A}_{\infty}-\mathrm{A}_{0}\right) \mathrm{e}^{-\beta \xi} \dot{\xi} \\
\dot{\mathrm{D}}=\beta\left(\mathrm{D}_{\infty}-\mathrm{D}_{0}\right) \mathrm{e}^{-\beta \xi} \dot{\xi} \\
\dot{\mathrm{G}}=\beta\left(\mathrm{G}_{\infty}-\mathrm{G}_{0}\right) \mathrm{e}^{-\beta \xi} \dot{\xi} \\
\dot{\xi}=\left\|\dot{\varepsilon}^{\mathrm{p}}\right\|
\end{gathered}
$$

Initial state : $\quad \xi=0, A=A_{0}, D=D_{0}, G=G_{0}$

Numerical Values : $\quad \beta=1000$

$$
\begin{array}{lll}
\mathrm{A}_{0}=0.454, & \mathrm{D}_{0}=0, & \mathrm{G}_{0}=1.35 \mathrm{MPa} \\
\mathrm{A}_{\infty}=0.608, & \mathrm{D}_{\infty}=1.07 \mathrm{MPa}, & \mathrm{G}_{\infty}=3.73 \mathrm{MPa}
\end{array}
$$


Even for 2D modelling, under plane strain or plane stress assumptions, numerical codes usually use 3D constitutive laws. Elaboration of a real 3D constitutive law requires specific investigations and simulation of 3D loading paths. However, it would be possible, thanks to simplified assumptions, to give a 3D extension of the law elaborated above. This extension would not be unique, but it would be very useful for implementation in numerical codes and for modelling of underground structures.

It is worthy of mention that in the model defined above, y axis corresponds to the vertical direction in the rock mass. This axis will be denoted by $\mathrm{z}$ in the 3D model. Then it will be assumed that the rock mass has isotropic properties in horizontal planes : all the horizontal directions are equivalent. Therefore an attempt is made to give a 3D expression in which $\mathrm{x}$ and $\mathrm{y}$ directions (horizontal directions) have equivalent properties. Moreover, the 3D law must give exactly the same 2D law for the plane stress case : substituting $\sigma_{\mathrm{y}}=0$ in this expression, the same expression (11) for the yield function must be found with y replaced by $\mathrm{z}$. To obtain this 3D expression one can proceed as following: $\left(\sigma_{\mathrm{xx}}-\sigma_{\mathrm{yy}}\right)$ in (8) is substituted first by $\left(\sigma_{\mathrm{xx}}-\sigma_{\mathrm{zz}}\right)$ (substitution of y axis by z) and then by $\left[\left(\sigma_{\mathrm{xx}}+\sigma_{\mathrm{yy}}\right) / 2-\sigma_{\mathrm{zz}}\right]$ (symetrisation of $\mathrm{x}$ and $\mathrm{y}$ directions). In the same way $\sigma_{\mathrm{xy}}$ in (11) is substituted first by $\sigma_{\mathrm{xz}}$ and then by $\left(\sigma_{\mathrm{xz}}+\sigma_{\mathrm{yz}}\right) / 2$. The $\sigma_{\mathrm{m}}$ is defined by $\sigma_{\mathrm{m}}=\left(\sigma_{\mathrm{xx}}+\sigma_{\mathrm{yy}}+\sigma_{\mathrm{zz}}\right) / 3$, and in order to obtain the same 2D expression for the case of plan stresses $\left(\sigma_{\mathrm{y}}=0\right), A \sigma_{\mathrm{m}}$ in (11) is replaced by $3 A \sigma_{\mathrm{m}} / 2$.

The final expression obtained for the $3 \mathrm{D}$ law is :

$$
f(\sigma, \xi): \sqrt{\left[\frac{1}{2}\left(\sigma_{x z}+\sigma_{y z}\right)-D\right]^{2}-(s-D)^{2}}-\sqrt{\left(-\frac{3}{2} A \sigma_{m}+G\right)^{2}+D^{2}}
$$

with :

$$
\mathrm{s}=\left[\left(\sigma_{\mathrm{xx}}+\sigma_{\mathrm{yy}}\right) / 2-\sigma_{\mathrm{zz}}\right] / 2, \quad \sigma_{\mathrm{m}}=\left(\sigma_{\mathrm{xx}}+\sigma_{\mathrm{yy}}+\sigma_{\mathrm{zz}}\right) / 3
$$

The values of the parameters $A, D, G$ and $\beta$ are exactly defined by the same relationships as in the 2D case.

The 3D constitutive model obtained in this way can be introduced in numerical codes in order to study stress and strain fields around underground structures in a rock mass under study. Indeed, such a modelling can be applied in case the characteristic dimension of the structure is greeter than that of the REV. In this case, stress and strain fields can be calculated using the homogenized behaviour model. Only, such a constitutive model is introduced in the numerical code and not the boundary conditions which are used for the computations which one made for the specific purpose of elaboration of such a model. 


\section{CONCLUSION AND PERSPECTIVES}

The method presented above allows to elaborate a homogenized constitutive model for a rock mass with a high density of fractures. This method is based on a few simplifying assumptions. Its very interesting feature is its capability to take account of the whole available data and information on the rock mass : distribution of fractures and constitutive behaviours of fractures and of intact rock. The authors believe that the suggested methodology provides a much more accurate model than more or less empirical models usually proposed in the framework of "rock mass classification». An interesting prospect and application of the present work would be to determine the strength properties of typical configurations of fractured rock masses in order to provide classification tables based on numerical calculations instead of empirical estimations. This may require a significant numerical work and a high number of long calculations. Nevertheless, such an effort may provide a very helpful tool for the estimation of fractured rock mass strength and for the prediction of damage risks and the related hydromechanical disturbances around underground facilities.

\section{REFERENCES}

1. F. Coste 'Comportement Thermo-Hydro-Mécanique des massifs rocheux fracturés', Thèse de Doctorat, Ecole Nationale des Ponts et Chaussées, Paris 1997.

2. N. Barton, R. Lien and J. LUNDE 'Engineering classification of rock masses for the design of tunnel support', Rock Mechanics, vol. 6, n 4, 1974, pp. 189-236.

3. Z.T. Bieniawski 'Rock mass classifications in rock engineering', Proceedings of the symposium on Exploration for Rock Engineering, Johannesburg November 1976.

4. P. de Buhan and S. Maghous 'Comportement élastique non linéaire macroscopique d'un matériau comportement un réseau de joints’, C.R. Acad. Sci. Paris, t. 324, Série II b, 1997, pp. 209-218.

5. A. Bekaert A. and S. Maghous 'Three-dimensional yield strength properties of jointed rock mass as a homogenized medium', Mechanics of cohesive frictional materials, vol. 1, 1996, pp. 1-24.

6. M. Kachanov 'Continuum model of medium with cracks', Proc. of ASCE, Engrg. Mech. Div., 1994, pp. 1039.

7. M. Cai and H. Horii 'A constitutive model of highly jointed rock masses', Mech. of Mat., Vol. 13, 1992, pp. 217-246.

8. M. Cai and H. Horii 'A constitutive model and FEM analysis of jointed rock masses', Int. J. Rock Mech. Min. Sci. \& Geomech. Abstr.; Vol. 30, No 4, 1993, pp. 352-359.

9. M. Le Ravalec and Y. Guéguen 'High and low frequency elastic moduli for a saturated porous/cracked rock - Differential self consistent and poro-elastic theory’, Geophysics, Vol. 61, No 4, 1996, pp. 1080-1094.

10. M. Oda 'A method for evaluating crack geometry on the mechanical behaviour of cracked rock masses’, Mechanics of Materials, Vol. 2, 1988, pp.163-171.

11. R. Budianski and R.J. O’Connell 'Elastic Moduli of cracked solid, Int. J. Solids Struct., Vol. 12, pp. 1976, 81-97. 
12. Z. Hashin 'Analysis of composite materials', J. App. Mech., Vol. 50, 1983, pp. 481-505.

13. C. Huet 'Application of variational concepts to size effects in elastic heterogeneous bodies', J. Mech. Phys. Solids, vol 38, No 6, 1990, pp. 813-841

14. J.C.S. Long, J.S. Remer, C.R. Wilson, P.A. Withespoon, 'Porous media equivalent for networks of discontinuous fractures’, Water Resources Research, Vol. 18, n 3, 1982, pp. 645-658.

15. J.C.S. Long and P.A. Withespoon 'The relation of the degree of interconnection to permeability in fracture networks', J. Geophys. Res., Vol. 90, n B4, 1985, pp. 3087-3098.

16. D.Billeux, J.P. Chiles, K. Hestir, J. Long 'Three dimensional statistical modelling of a fractured rock mass - An example from the Fanay-Augères mine, Int. J. Rock Mech. Min. Sci., \& Geomech. Abstr., Vol. 26, n 3-4, 1989, pp. 281-299.

17. R.E. Goodman 'Methods of geological engineering in discontinuous rocks', West Publishing Company, 472p., 1976.

18. R. Hill, J. Mech. Phys. Solids, 1963, Vol. 11, p. 357.

19. R. Hill 'The essential structure of constitutive structure of constitutive laws for metal composites and polycrystals', J. Mech. Phys. Solids, 1967, Vol. 15, pp. 70-95.

20. D. François, A. Pineau, A. Zaoui 'Elasticité et Plasticité', Editions Hermes, Paris 1992, pp.77-79. 